\title{
Bilateral Geographic Atrophy of the Retina in Acquired Immune Deficiency Syndrome Associated with Cryptococcal Meningitis
}

\author{
${ }^{1} 251$ General Air-Force Hospital, Athens, Greece \\ ${ }^{2}$ Tzaneio Prefecture General Hospital of Piraeus, Piraeus, Greece \\ ${ }^{3}$ Glyfada's Regional Health Unit, Social Insurance Institute, Athens, Greece. \\ ${ }^{4}$ National Technical University of Athens, School of Chemical Engineering, Athens, Greece \\ *Corresponding author: Emmanouil Alexakis, School of Chemical Engineering, Greece
}

Evangelia Chalkiadaki ${ }^{1}$, Michail Tzortzakakis ${ }^{2}$, Chrysoula Florou ${ }^{3}$ and Emmanouil Alexakis ${ }^{4 *}$

\begin{tabular}{|c|c|}
\hline ARTICLE INFO & ABSTRACT \\
\hline 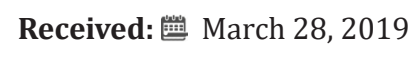 & Cryptococcal infections may occur in $5-10 \%$ of patients with AIDS and are associated \\
\hline Published: 蔧 April 04, 2019 & with both direct and indirect ocular complications. It may result in meningitis and sec- \\
\hline
\end{tabular}
eral Geographic Atrophy of the Retina in Acquired Immune Deficiency Syndrome Associated with Cryptococcal Meningitis. Biomed J Sci \& Tech Res 16(5)-2019. BJSTR. MS.ID.002906.

Keywords: Cryptococcal Meningitis; Geographic atrophy

Abbreviations: CMV: Cytomegalovirus; CF: Counting Fingers; OCT: Optical Coherence Tomography; RPE: Retinal Pigment Epithelium

\section{Introduction}

Ocular manifestations are found in the majority of patients with AIDS. The most common ocular finding is a noninfectious occlusive microangiopathy known as HIV retinopathy. Nevertheless, visually more significant and devastating are opportunistic ocular infections such as cytomegalovirus (CMV) retinitis (the most common), progressive retinal necrosis, acute retinal necrosis, bacterial, fungal and protozoal retinochoroiditis [1]. Fungal ocular infections include Pneumocystis choroiditis, Histoplasma chorioretinitis, Cryptococcal choroiditis and Candidiasis. There seems to be a distinction between those diseases that have the potential to affect both eyes of HIVpatients, such as CMV retinitis, acute retinal necrosis, progressive outer retinal necrosis, HIV-related ischaemic microvasculopathy, ocular syphilis, ocular tuberculosis, cryptococcal meningitis, ocular toxic or allergic drug reactions and disorders which tend to occur unilaterally. Ocular toxoplasmosis, Kaposi's sarcoma of the ocular adnexae and conjunctival neoplasias tend to affect one eye only [2]. Cryptococcus is the most common life-threatening fungal pathogen that affects patients with HIV infection [2]. It is an encapsulated, basidiomycetic yeast, commonly found in pigeon excreta and rotten wood. The yeast causes Cryptococcosis, an infectious disease with worldwide distribution and wide array of clinical presentations. Approximately $95 \%$ of cryptococcal infections are caused by Cryptococcus neoformans [3]. It is a well-known infection in immunocompromised individuals but can occasionally occur in immunocompetent individuals as well. It enters the body through inhalation and can spread to the eye in the bloodstream or from the CNS via the optic nerve. It has a propensity to seed the central nervous system during hematogenous dissemination and can result in a severe, chronic form of meningitis. Occasionally, the resulting meningitis is further complicated by visual loss.

In undeveloped areas, loss of visual acuity is a relatively common occurrence and is seen in up to $10 \%$ of patients with AIDS and Cryptococcus infection. The etiology of visual complications associated with cryptococcal meningitis remains unclear. Possible explanations for visual loss include direct infiltration of the optic nerve or optic tracts by the fungus, inflammatory compression of the optic nerve, adhesive arachnoiditis, amphotericin B toxicity used as a therapeutic regimen, cerebral vasculitis, intracranial 
hypertension and very rarely cryptococcal choroiditis [4]. We present a case of an HIV-positive patient with bilateral, permanent visual loss complicating cryptococcal meningitis.

\section{Case Report}

A 51-year-old Caucasian HIV-positive woman presented to our clinic complaining of decreased vision in both eyes for the last 10 years. She was diagnosed with HIV infection 20 years ago and was hospitalized for cryptococcal meningitis 10 years ago. She mentioned that, at that time, her CD $4+$ count was 40 cells/ $\mu \mathrm{L}$. Upon examination, visual acuity was counting fingers (CF) $2 \mathrm{ft}$ in both eyes and intraocular pressure was $14 \mathrm{~mm} \mathrm{Hg}$ bilaterally by noncontact tonometry. The anterior segment examination in both eyes was unremarkable, but fundoscopy revealed geographic atrophy and pigment deposition in the macula and the mid- periphery of the fundus of both eyes (Figure 1). We performed an optical coherence tomography (OCT) which showed atrophy of the retinal layers in the macula (Figure 2). We also performed fundus autofluorescence which showed retinal pigment epithelium (RPE) atrophy (Figure
3) and fluorescein angiography which revealed multiple window defects in the macula and the mid-periphery of the fundus of both eyes corresponding to the retinochoroidal atrophy, with no sign of active disease (Figure 4). We established the diagnosis of geographic atrophy of the retina due to a past retinitis. The differential diagnosis of retinitis included VZV retinitis, HSV retinitis, CMV retinitis, Cryptococcal retinitis, ocular syphilis and Tb retinochoroiditis. We asked for a blood examination and a serology test for HSV-1 and HSV-2 (IgG, IgM), VZV (IgG, IgM), CMV (IgG, IgM), VDRL, FTA-ABS, RPR and Quantiferon-TB Gold test. We also performed a Mantoux tuberculin test and a Chest X-ray. All our results were negative for a past herpetic, cytomegalovirus, syphilitic or tuberculosis infection. At the time of our examination, her CD4+ count was 430 cells $/ \mu \mathrm{L}$, she was in a pretty good general health and was under HAART. Due to the medical history of cryptococcal meningitis and the fact that the patient mentioned that she was first experiencing an alteration in visual acuity two months after her hospitalization 10 years ago, we assumed that Cryptococcus was the cause for the geographic atrophy of the retina.
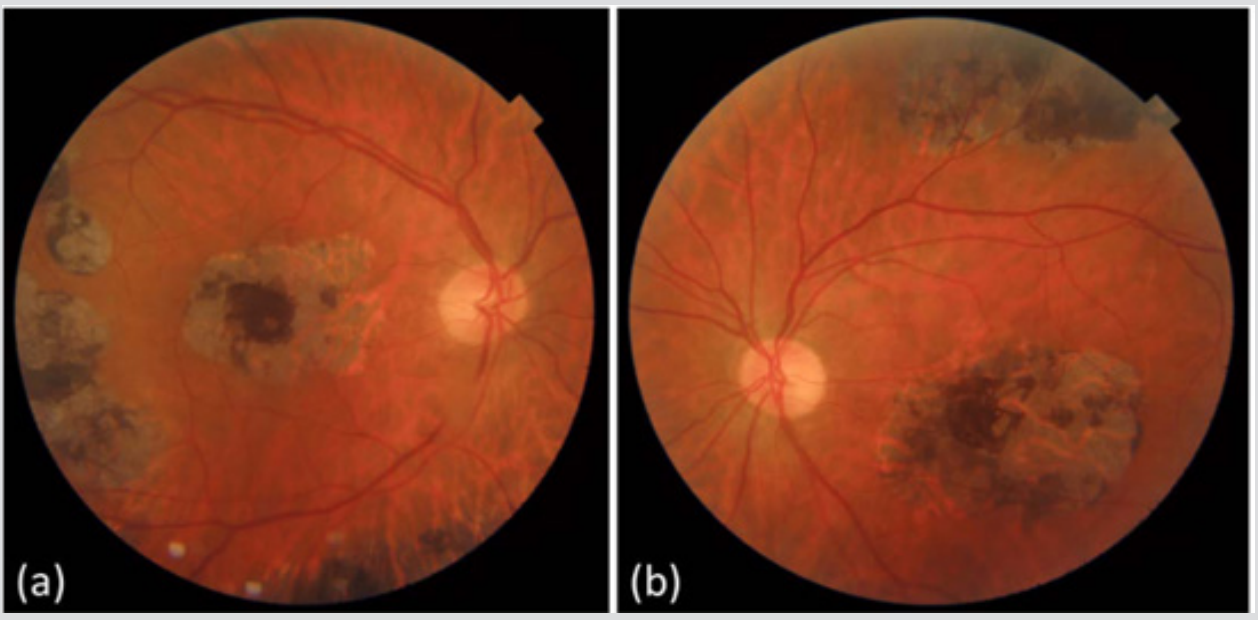

Figure 1: Colour fundus photography of the Right (a) and the Left (b) eye, showing geographic atrophy and pigment deposition in the macula and mid-periphery of the patient.

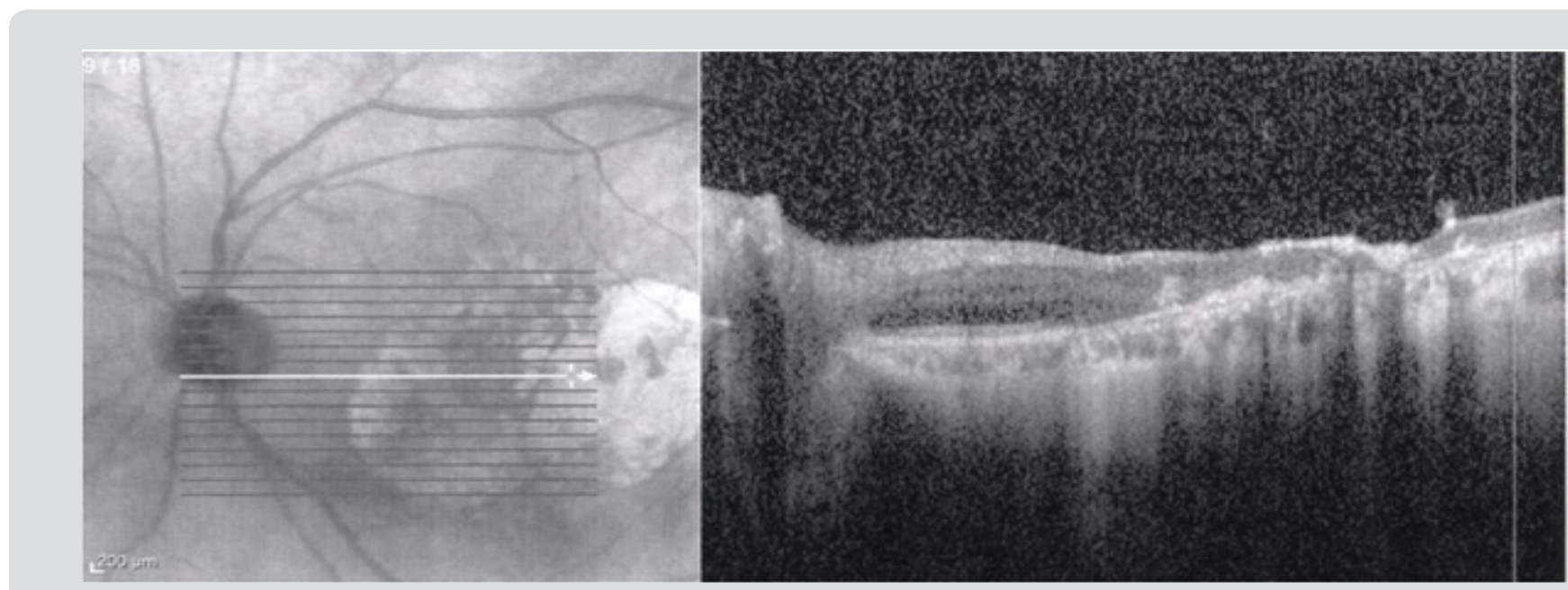

Figure 2: Optical Coherence Tomography (OCT) of the left eye showing retinal atrophy in the macula. 

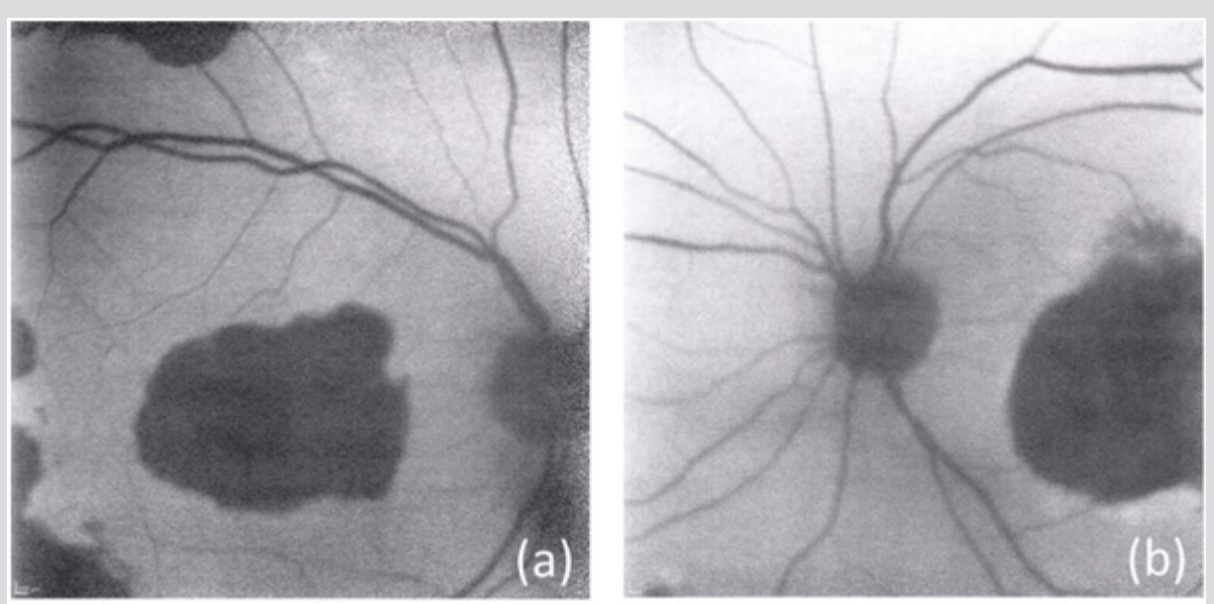

Figure 3: Fundus autofluorescence (FAF) of the Right (a) and the Left (b) eye showing atrophy of the retinal pigment epithelium (RPE) in the macula and the mid-periphery.
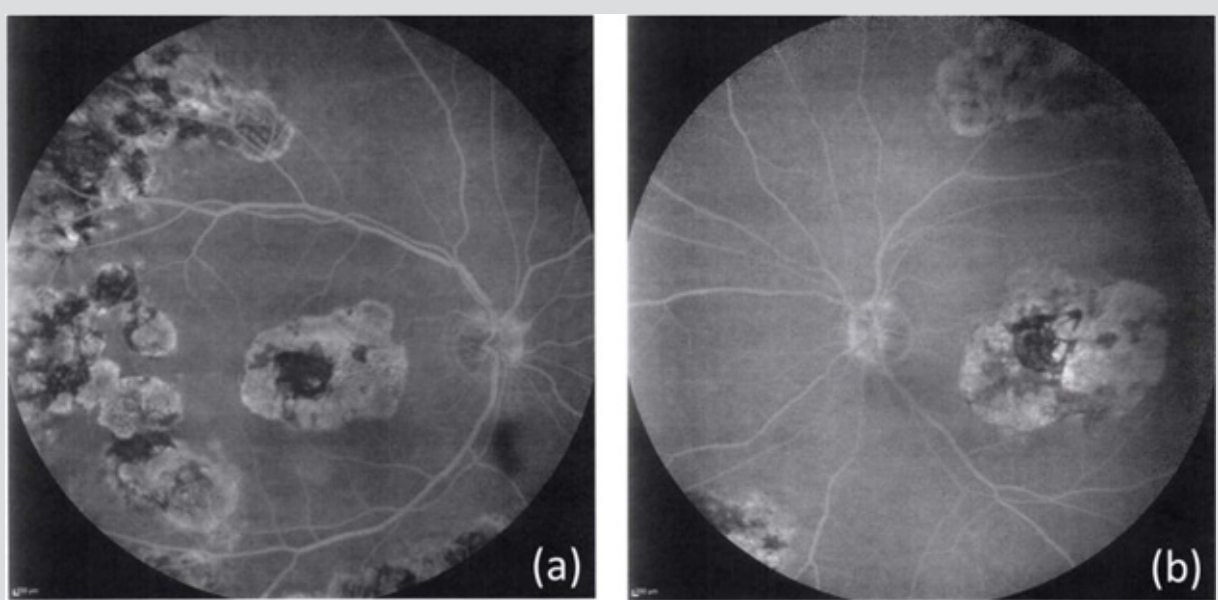

Figure 4: Fluorescein angiography (FA) of the Right (a) and the Left (b) eye showing multiple window defects corresponding to the patches of chorioretinal atrophy.

We established the diagnosis of geographic atrophy of the retina due to a past retinitis. The differential diagnosis of retinitis included VZV retinitis, HSV retinitis, CMV retinitis, Cryptococcal retinitis, ocular syphilis and $\mathrm{Tb}$ retinochoroiditis. We asked for a blood exam and a serology test for HSV-1 and HSV-2 (IgG, IgM), VZV (IgG, IgM), CMV (IgG, IgM), VDRL, FTA-ABS, RPR and QuantiferonTB Gold test. We also performed Mantoux test and a Chest X-ray. All our results were negative for a past herpetic, cytomegalovirus, syphilitic or tuberculosis infection. At the time of our examination, her $C D 4^{+}$count was 430 cells $/ \mu \mathrm{L}$, she was in a pretty good general health and was under HAART. Due to the medical history of cryptococcal meningitis and the fact that the patient mentioned that she was first experiencing an alteration in visual acuity two months after her hospitalization 10 years ago, we assumed that Cryptococcus was the cause for the geographic atrophy of the retina.

\section{Discussion}

Cryptococcus neoformans is a fungal pathogen that causes almost half a million deaths each year. Cryptococcal meningitis preferentially occurs in people with impaired cell-mediated immunity and is a major HIV-related opportunistic infection occurring when the $\mathrm{CD} 4^{+}$cell count falls below 100 cells/ $\mu \mathrm{L}$. With widespread implementation of successful antiretroviral therapy (HAART), the incidence of HIV-associated cryptococcosis has decreased significantly in most developed countries, although the incidence in other at-risk populations has not changed [3]. There have been found multiple yeast virulence factors of Cryptococcus neoformans. The 3 classical and most prominent factors include capsule formation, melanin pigment production, and thermotolerance [5].

Ocular signs and symptoms such as ocular palsies and papilloedema have been noted in a substantial proportion of cases of cryptococcal meningitis, even before the AIDS epidemic [6]. Several other ocular manifestations of cryptococcosis have been identified since then. Ocular involvement may occur directly with vasculitis and retinal exudate, such as in multifocal choroiditis, or more commonly indirectly with papilloedema and ocular motility dysfunction. Visual loss can also be the result of endophthalmitis 
due to extensive retinal disease with or without vitritis [7]. In some cases, visual loss may be owing to optic nerve infiltration by yeasts and cryptococcomas in the visual pathway, including the optic nerve, chiasm and tract. Irreversible blindness may be also due to vascular compromise of the optic nerve from intracranial hypertension [3]. There seem to be two patterns of visual loss in immunodeficient patients, one with the rapid onset of visual loss with limited effective treatment options, and the other with gradual visual deterioration. This difference probably relies on the different etiologic mechanisms. The rapid onset of vision loss (3 days) is thought to result from direct infiltration of the optic nerve or adhesive or inflammatory arachnoiditis $[8,9]$, while visual loss over a period of more than four weeks has been attributed more commonly to long term intracranial hypertension [9].

Our patient presented 10 years after her visual acuity started to decline, limiting our capability for a certain diagnosis. She suffered permanent, bilateral visual loss due to bilateral geographic atrophy. There were found extensive, multiple foci of retinal atrophy both in the macula and the periphery of the fundus of both eyes indicating a past retinal infection. There was no sign of reactivation of the disease. Serological tests were negative for many possible causative factors such as herpetic infection. Taking into consideration her past medical history, we regarded this to be a case of past cryptococcal chorioretinitis. Unfortunately, there was no therapeutic regimen to be done by the time of presentation to our clinic.

\section{References}

1. DA Jabs (1995) Ocular manifestations of HIV infection. Trans Am Ophthalmol Soc 93: 623-683.

2. Philippe G Kestelyn, Emmett T Cunningham Jr ((2001) HIV/AIDS and blindness. Bull World Health Organ 79(3): 208-213.

3. Eileen K Maziarz, John R Perfect (2016) Cryptococcosis. Infect Dis Clin North Am 30(1): 179-206.

4. J Duggan, HM Walls (2012) Ocular Complications of Cryptococcal Meningitis in Patients with HIV: Report of Two Cases and Review of the Literature. J Int Assoc Physicians AIDS Care (Chic) 11(5): 283-288.

5. Coelho C, Bocca AL, Casadevall A (2014) The tools for virulence of 4. Adv Appl Microbiol 87: 1-41.

6. Okun E, Butler WT (1964) Ophthalmologic complications of cryptococcal meningitis. Arch Ophthalmol 71: 52-57.

7. Rex JH, Larsen RA, Dismukes WE, Cloud GA, Bennett JE (1993) Catastrophic visual loss due to Cryptococcus neoformans meningitis. Medicine (Baltimore) 72(4): 207-224.

8. Cohen DB, Glasgow BJ (1993) Bilateral optic nerve cryptococcosis in sudden blindness in patients with acquired immune deficiency syndrome. Ophthalmology 100(11): 1689-1694.

9. Bach MC, Tally PW, Godofsky EW (1997) Use of cerebrospinal fluid shunts in patients having acquired immunodeficiency syndrome with cryptococcal meningitis and uncontrollable intracranial hypertension. Neurosurgery 41(6): 1280-1282.

\section{ISSN: 2574-1241}

DOI: 10.26717/BJSTR.2019.16.002906

Emmanouil Alexakis. Biomed J Sci \& Tech Res

This work is licensed under Creative Commons Attribution 4.0 License

Submission Link: https://biomedres.us/submit-manuscript.php

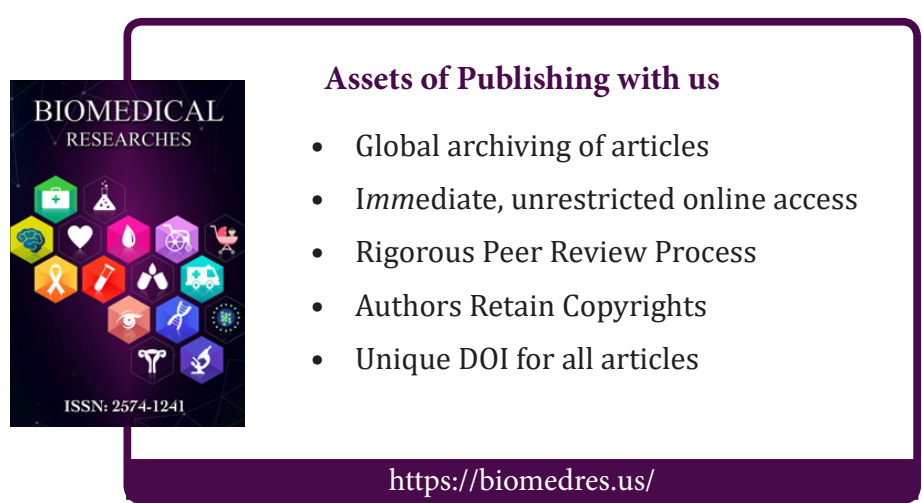

